\title{
Synthesis of SAPO-20 Using Isopropylamine as a Template from a SAPO-34 Precursor
}

\author{
Hairong ZHANG ${ }^{\mathrm{a}}$, Hongyan $\mathrm{LIU}^{\mathrm{a}, 1}$, Ruijie BAI ${ }^{\mathrm{a}}$, Rong SUN ${ }^{\mathrm{a}}, \mathrm{Xi} Z \mathrm{ZHANG}^{\mathrm{a}}$, Xinyi XIE ${ }^{\mathrm{a}}$, \\ Feilong YU ${ }^{\mathrm{a}}$, Yu JIANG ${ }^{\mathrm{b}}$ and Yong GUO ${ }^{\mathrm{a}, 2}$ \\ ${ }^{a}$ College of Chemistry and Chemical Engineering, Institute of Applied Chemistry, \\ ShanXi Datong University, Datong 037009, China \\ b Datong Coal Mine Group Co. LTD, Datong 037003, China
}

\begin{abstract}
SAPO-20 molecular sieve with the SOD framework was successfully synthesized using SAPO-34 as the precursor and isopropylamine (IPA) as the template by phase- transformation route. The influence of post-treatment time (in IPA, $\mathrm{H}_{2} \mathrm{O}$, and SAPO-34 system) and $\mathrm{NaOH}$ concentration (in IPA, $\mathrm{NaOH}, \mathrm{H}_{2} \mathrm{O}$, and SAPO-34 system) on the products was investigated. The results showed that sheet debris SAPO-34 building the hollow-inside cubic morphologies which was padding with irregular SAPO-34. It was received for the as-synthesized spherical

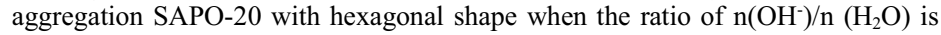
0.12 and the ratio of $n(I P A) / n\left(\mathrm{H}_{2} \mathrm{O}\right)$ is 0.07 in the synthesis gel. This method not only develops a new route for the synthesis of SAPO-20 molecular sieve, but also provides a way of using waste SAPO-34 catalyst, which can be used as a new exploration model for energy saving, consumption reduction and comprehensive utilization in SAPO-34 catalyst process.
\end{abstract}

Keyword. SAPO-34, SAPO-20, morphology; hollow-inside cubic, phase-transformation route

\section{Introduction}

Sodalite, AlPO-20 and SAPO-20 are analogs of the sodalith structure (SOD), that is, they have stacking sequence of $\mathrm{ABC}$ of 6-ring, cubic, $\operatorname{Im}^{3} \mathrm{~m}, \mathrm{a}=8.870 \AA$. The syntheses of the very small pore SAPO-20 with the pore sizes of $0.3 \mathrm{~nm}$ and the pore volumes of $0.40 \mathrm{~cm}^{3} / \mathrm{g}$ were described in the $1980 \mathrm{~s}[1-4]$.

There are many methods for the synthesis of SAPO-20, such as: hydrothermal crystallization method [1, 4-10], $\mathrm{F}^{-}$system hydrothermal synthesis [11, 12], dry-gel conversion method [13], and liquid-phase crystallization [14]. The silicon source for the synthesis of SAPO-20 molecular sieves is silica sol [1, 5, 7, 10-13], white carbon black $[5,6]$ and fumed silica $[8,9]$ while aluminum source is involved in aluminum isopropoxide [5], activate alumina [5, 13], pseudoboehmite [1, 5-7, 9, 10, 12], bayerite [4], aluminum hydroxide [8], and aluminium chloride [11].

${ }^{1}$ Corresponding Author, Hongyan LIU, College of Chemistry and Chemical Engineering, Institute of Applied Chemistry, ShanXi Datong University, Datong 037009, China; Email: liuhongyan9629@163.com.

2 Corresponding Author, Yong GUO, College of Chemistry and Chemical Engineering, Institute of Applied Chemistry, ShanXi Datong University, Datong 037009, China; Email: ybsy_guo@163.com. 
The interzeolite conversion method is an alternative synthesis strategy for the hydrothermal conversion of one zeolite into another [15-19]. To the best of our knowledge, this is the first reported on the transformation of CHA-type zeolite into SOD-type zeolite. It is well to know that SAPO-34 molecular sieve is a catalyst for the industrialized production of olefin from methanol. However, there are two major problems for SAPO-34 molecular sieve catalysts in the process of methanol conversion to olefins. One is that SAPO-34 is rapidly inactivated for carbon deposition because of its small pore size (8-ring, $0.43 \mathrm{~nm}$ ). Although improving the anti-coking of the nanosized SAPO-34 or hierarchical pore structure SAPO-34 catalyst by changing the synthesis conditions, it still can not fundamentally solve the problem of deactivation. The other is that SAPO-34 catalyst is easy to be worn out which recycled in fluidized bed reactor. The wear resistance of the catalyst can be improved to a certain extent by controlling the molding conditions or adding binders of the catalyst. After MTO industrialization, many cycles of regeneration and wear of the catalyst will result in a large amount of waste material containing silicon, aluminum and phosphorus. Reasonable and efficient use of the waste material can not only save resources, but also improve the economic benefits of MTO process [20].

This work is mainly focused on the two following respects: (1) the effects of post-treatment time of SAPO-34 molecular sieve in the presence of IPA and water on its structure, (2) the effect of $\mathrm{NaOH}$ concentration on the product selectivity. A novel method for low-cost synthesis of SAPO-20 molecular sieves using SAPO-34 molecular sieves and IPA as raw materials is presented.

\section{Experimental Section}

\subsection{Reagents}

The following starting materials were used for the synthesis: pseudo boehmite $(75 \%$ $\mathrm{Al}_{2} \mathrm{O}_{3}$ ), $\mathrm{NaOH}$ (Merc), phosphoric acid (85\%), CAB-O-SILM-5, TMAOH (25 wt $\%$ ), and isopropyl amine (IPA, Fluka). All chemicals were employed without further purification.

\subsection{Synthesis}

\subsubsection{Synthesis of SAPO-34}

Hydrothermal method was explored to prepare SAPO-34 which was reported in Ref. [21]. In a typical procedure for the synthesis of SAPO-34, $9.2 \mathrm{~g}$ of pseudoboehmite was slurried in $28 \mathrm{~g}$ of deionized water and $15.4 \mathrm{ml}$ of orthophosphoric acid and stirred for 7 hours. Then $6.5 \mathrm{~g} \mathrm{M}-5,11.6 \mathrm{~g}$ morpholine and $39 \mathrm{~g}$ water mixed well and slowly added to before-mentioned solution. The hydrogel stirred for another 7 hours with the following molar composition: $1.0 \mathrm{P}_{2} \mathrm{O}_{5}: 1.0 \mathrm{Al}_{2} \mathrm{O}_{3}: 1.1 \mathrm{SiO}_{2}: 1.3$ morpholine: $52 \mathrm{H}_{2} \mathrm{O}$. Crystallization was conducted at $200{ }^{\circ} \mathrm{C}$ for 4 days. After crystallization the product was filtrated from the mother liquor, washed with deionized water and dried at $100{ }^{\circ} \mathrm{C}$ for $12 \mathrm{~h}$. The as-synthesized product labeled as ST.

\subsubsection{Synthesis of SAPO-20}

Hydrothermal method was explored to prepare SAPO-20 which was reported in Ref. 
[2]. The molar ratio of the reaction mixture was 0.75TMAOH: $\mathrm{P}_{2} \mathrm{O}_{5}: \mathrm{Al}_{2} \mathrm{O}_{3}: \mathrm{SiO}_{2}$ : $50 \mathrm{H}_{2} \mathrm{O}$. In a typical synthesis, $2.8 \mathrm{~g}$ of orthophosphoric acid was added to $6.5 \mathrm{~g}$ of deionized water. Then $1.7 \mathrm{~g}$ of pseudoboehmite was slowly added in the phosphoric acid solution. The obtained mixture is aged for half of hour. To this mixture $2.1 \mathrm{ml}$ of silica sol was added and mixed well. $7.9 \mathrm{ml}$ of TMAOH was added after this was stirred for another three hours. The final mixture $\mathrm{pH}$ was about 7. Crystallization was conducted at $150{ }^{\circ} \mathrm{C}$ for 3 days. After crystallization the product was filtrated from the mother liquor, washed with deionized water and dried at $100{ }^{\circ} \mathrm{C}$ for $12 \mathrm{~h}$. The sample was labeled as SOD-R.

\subsubsection{Post-treatment Time}

A typical procedure for the post-treatment was carried out as follows. 0.85 grams of as-synthesized SAPO-34 was added to a solution consisting of $12.6 \mathrm{~g}$ of IPA mixed with $55 \mathrm{~g}$ of water under stirring. The resulting mixture was then transferred into an autoclave, which was heated to $160{ }^{\circ} \mathrm{C}$ and kept at this temperature for $1,2,3,4$ and 8 days, and the samples are labeled as T-1, T-2, T-3, T-4 and T-5, respectively. After crystallization, the solid product was recovered by filtration, washed with deionized water, and dried at $100{ }^{\circ} \mathrm{C}$ for $12 \mathrm{~h}$. As comparison, $7.9 \mathrm{~g}$ IPA was used, and the temperature was $160{ }^{\circ} \mathrm{C}$ and the post-treatment time was $4 \mathrm{~d}$. The sample was labeled as T-6.

\subsubsection{Phase-Transformation}

Synthesis of SAPO-20 from a SAPO-34 Precursor. A typical procedure for the phase-translation was carried out as follows. $0.85 \mathrm{~g}$ of as-synthesized SAPO-34 was added to a solution containing $12.6 \mathrm{~g}$ of IPA, $\mathrm{x}$ g sodium hydroxide and $55 \mathrm{~g}$ of water under stirring $(\mathrm{x}=0.5 \mathrm{~g}, 1.0 \mathrm{~g}$ and $1.5 \mathrm{~g})$. The resulting mixture was then transferred into an autoclave, which was heated to $160{ }^{\circ} \mathrm{C}$ and kept at this temperature for 4 days, and the samples are denoted as $\mathrm{C}-1, \mathrm{C}-2$, and $\mathrm{C}-3$, respectively. After crystallization, a series of operation, such as filtrating, washing with distilled water and drying in air, was carried out and finally the solid product was recovered. As comparison, $0.85 \mathrm{~g}$ of $\mathrm{T}-6,12.6 \mathrm{~g}$ of IPA, $1.5 \mathrm{~g}$ sodium hydroxide and $55 \mathrm{~g}$ of water were used. The sample was labeled as C-4.

\subsection{Characterization}

It was performed for X-ray diffraction (XRD) on a Rigaku D/max-2500-type powder diffractometer with Ni-filter $(\mathrm{Cu} \mathrm{K} \alpha$ radiation $\lambda=0.15418 \mathrm{~nm})$. The diffraction pattern was recorded within the angular range of $2 \theta$ from 5 to $35^{\circ}$ with an interval of $0.5 \mathrm{~s}$ and steps of $0.02^{\circ}$ at $30 \mathrm{~mA}$ (tube current) and $40 \mathrm{kV}$ (tube voltage). The morphology and size of the products were characterized by a Jeol 6300-F scanning electron microscope (SEM, Shimadzu Corporation) operated at 10-15 kV.

\section{Results and Discussion}

\subsection{Effect of Post-treatment Time on SAPO-34 Structure}

The XRD pattern of as-synthesized ST (figure 1 ST) is in agreement with that of 
SAPO-34 molecular sieve [2], and no other peaks were detected. This implies that pure SAPO-34 is obtained. The effect of time of IPA post-treatment SAPO-34 on its structure are shown in figure 1 . We can see from figure 1, post-treatment time from T-1 to $\mathrm{T}-5$, the products maintain the $\mathrm{CHA}$ structure. Prolonging the post-treatment time, the XRD diffraction peaks intensity decreases. Moreover, there are three weak peaks at $2 \theta=7.1,19.5$ and $21.4^{\circ}$ in T-5, which indicating that some of SAPO-5 has been formed. For sample T-6, the post-treatment product is amorphous, which indicating that IPA is good for the stabilization of SAPO-34 structure.

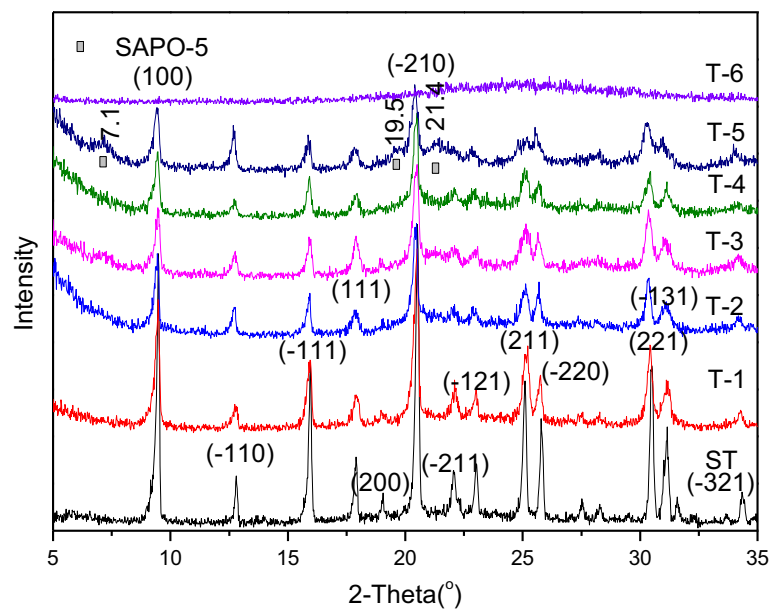

Figure 1. XRD pattern of ST, T-1, T-2, T-3, T-4, and T-5.

In addition, it was observed that post-treatment time strongly governs the morphology and the size of the crystals. Figure 2 shows the SEM images of sample ST-T-5. It is obvious that the above samples have completely different morphologies. Sample ST with pure phase CHA was observed the typical cubic morphology which is $6-10 \mu \mathrm{m}$ in size. Samples T-1 show different morphologies with sheet debris, irregularity, cubic, defective cubic, and defective hollow-inside cubic. Prolonged post-treatment time from T-2 to T-5, the cubic SAPO-34 disappears, the sheet debris SAPO-34 is depleted, and the size of irregularity materials are decreased. Moreover, for sample T-5, there are cylindrical or spherical products which is SAPO-5 by XRD.

In our previous study [20], we discovered that the $\mathrm{OH}^{-}$concentration had a great effect on the product selectivity fixed the post-treatment conditions. The evolution of products with increasing the $\mathrm{OH}^{-}$concentration were low crystallization SAPO-34, SAPO-34 and SAPO-40, and SAPO-25 (or AlPO-25) and SAPO-56. 

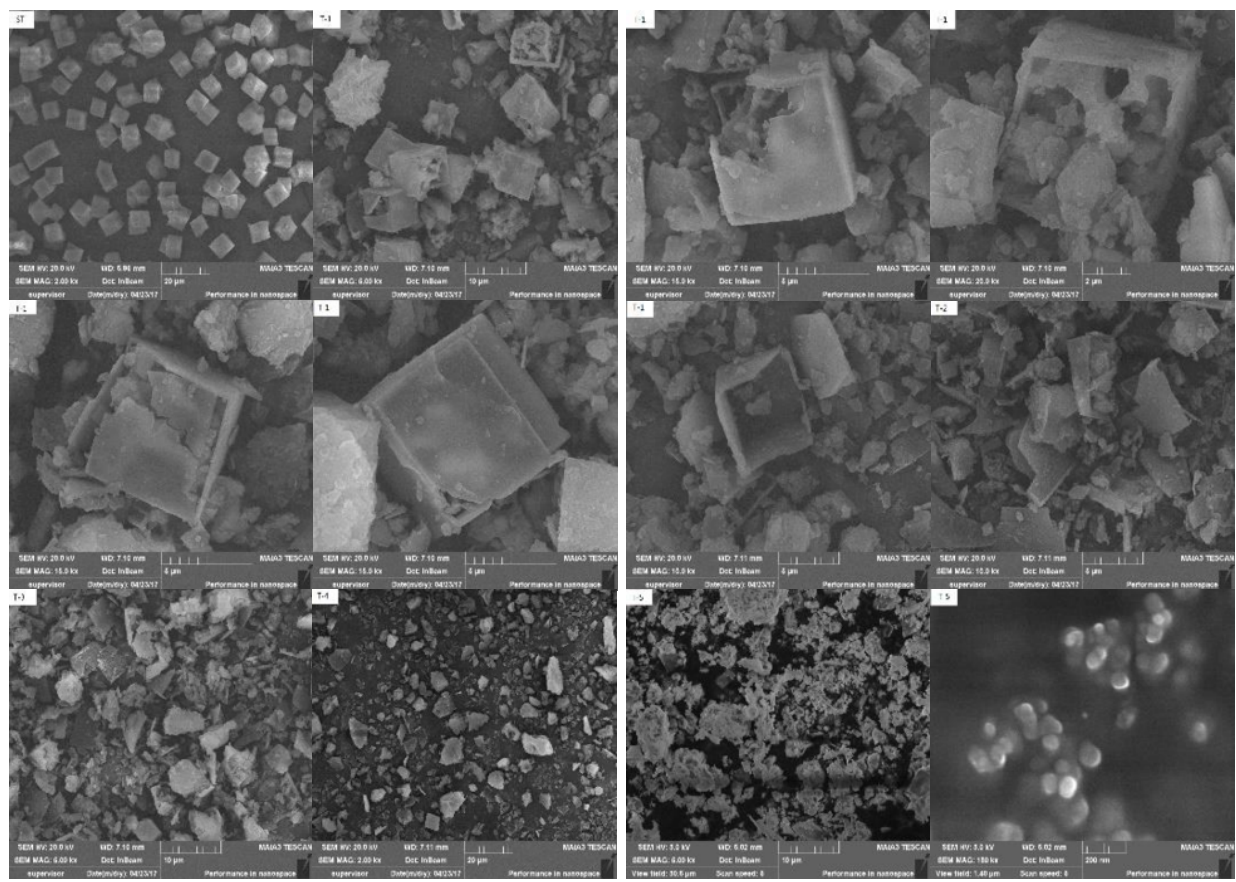

Figure 2. SEM images of ST, T-1, T-2, T-3, T-4, and T-5.

\subsection{Effect of NaOH Concentration on the Product Selectivity}

The XRD pattern of SOD-R (figure 3) is in agreement with that of SAPO-20 [2], which the $2 \theta$ values at $13.9,19.7,22.0,24.2,27.9,31.3$ and $34.4^{\circ}$. The effect of $\mathrm{NaOH}$ concentration on the product of phase-transformation was investigated with the gel molar composition of $x \mathrm{NaOH}: 21$ IPA:350 $\mathrm{H}_{2} \mathrm{O}$ (and $0.85 \mathrm{~g}$ SAPO-34 (ST) or $0.85 \mathrm{~g}$ (T-6)). The XRD patterns of obtained samples are shown in figure 3 . Without $\mathrm{NaOH}$, the product is CHA with low crystallization (figure $1 \mathrm{~T}-4$ ). At low concentrations of $\mathrm{x}=12.5(\mathrm{C}-1)$, the main product is SAPO-20 molecular sieve, but it co-crystallized with SAPO-2 1 corresponding the $2 \theta$ values at 15.7, 17.3, 18.2, 18.9, 20.6, 21.2, 21.6, 25.9, $26.3,28.6,30.6$, and $32.6^{\circ}$ [2]. However, when the $\mathrm{NaOH}$ amount was increased to $\mathrm{x}=25$ (C-2), the SAPO-21 content was increased. At $\mathrm{x}=37.5(\mathrm{C}-3$ and $\mathrm{C}-4)$ pure SAPO-20 crystal can be observed. Moreover, with increasing the amount of sodium hydroxide, the $2 \theta$ values of the characteristic peaks of SAPO-20 shift to a low angle.

Figure 4 shows the morphology of the products. SOD-R (SAPO-20 molecular sieves, illustrated in figure 4) has spherical morphology with particle size varying between 1 to $5 \mu \mathrm{m}$. C-1 (a mixture of SAPO-20 and -21) display irregular shapes, while C-3 exhibits hexagonal shape (SAPO-20) and cylindrical morphology (SAPO-21). The morphology of C-4 with spherical aggregation consisted of hexagonal shape SAPO-20 is quite different from SOD-R. 


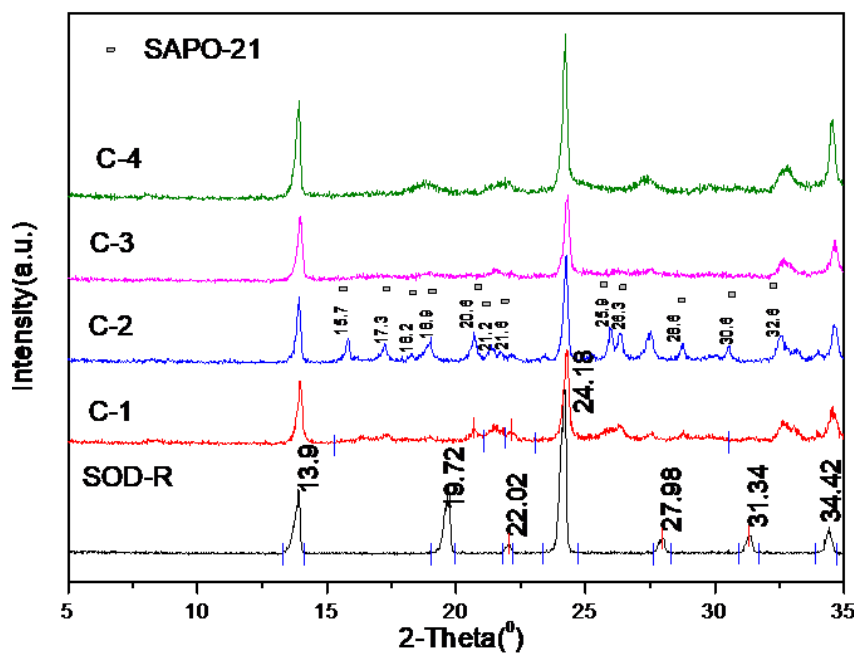

Figure 3. XRD pattern of SOD-R, C-1, C-2, C-3, and C-4.

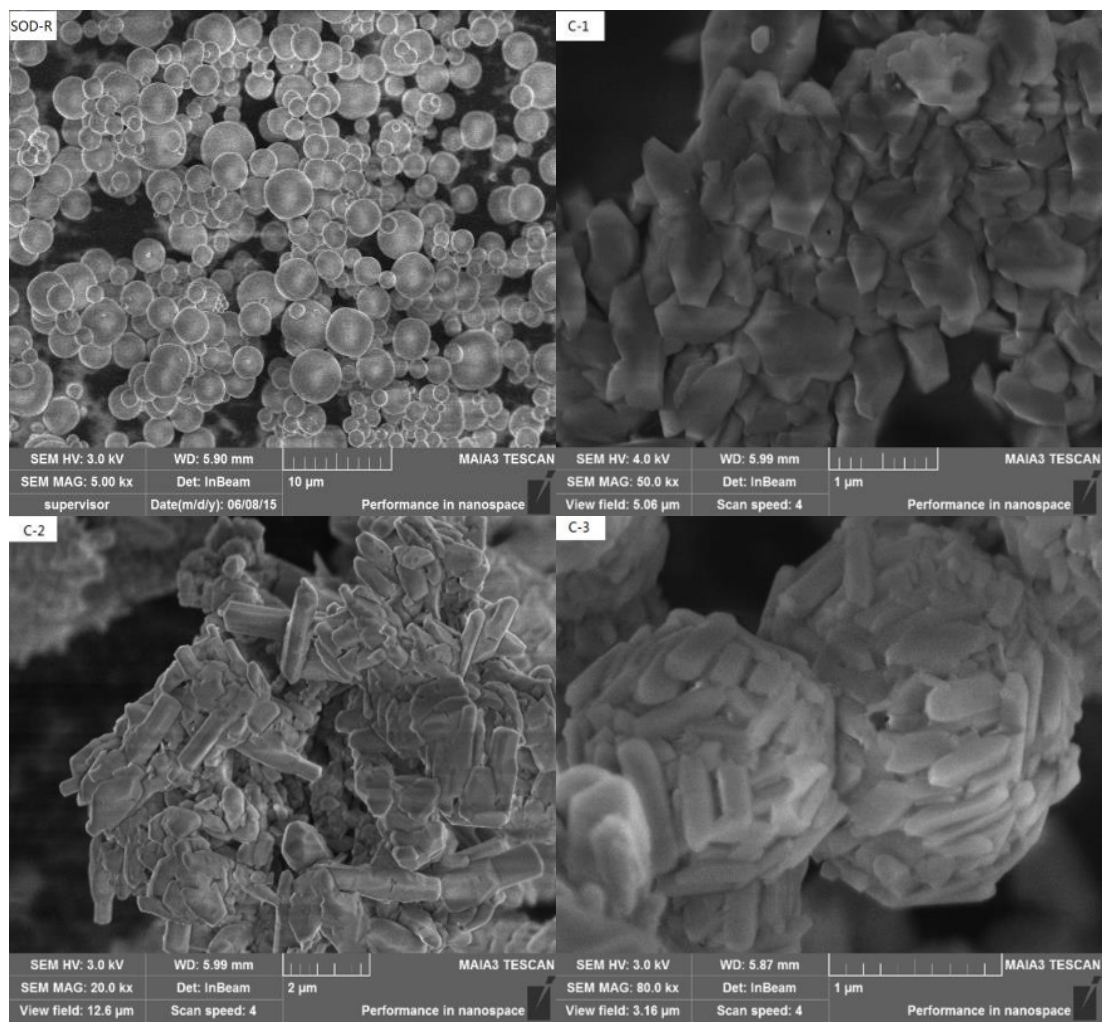

Figure 4. SEM images of SOD-R, C-1, C-2, C-3, and C-4. 


\section{Conclusion}

A SAPO-20 silicoaluminophosphate molecular sieve with very small-pore has been synthesized using IPA template and spent SAPO-34 as starting materials in aqueous media without addition of any external silicon source and aluminum source. This work has certain reference significance for the generation of cubic morphology of SAPO-34 molecular sieves.

\section{Acknowledgments}

This work has been supported by the Research Project supported by Shanxi Scholarship council of China (2016-104), Datong Science and Technology Public Relations Project $(2015021,2018023)$, University Students Innovation and Entrepreneurship of Shanxi Datong University (XDC2017257) and the Doctoral Science Foundation of ShanXi DaTong University (No. QD201049). We also thank HongJie Li, FeiFei Sun, and QiYi Bu for their assistance with the reaction experiments.

\section{References}

[1] Li H Y, Liang J, Wang Z W, Liu Z M, Huang X Y and Zhao S Q 1987 Synthesis and thermal stability of SAPO molecular sieves Nat. Gas Chem. Ind. 121.

[2] Lok B M, Messina C A, Patton R L, Gajek R T, Cannan T R and Flanigen E M 1984 Crystalline Silicoaluminophosphates US Patent 4440871.

[3] Lok B M, Messina C A, Patton R L, Gajek R T, Cannan T R and Flanigen E M 1984 Silicoalumino phosphate molecular sieves:another new class of microporous crystalline inorganic solids Am. Chem. Soc $\mathbf{1 6 0} 6092$.

[4] Xu W, Dou T and Wu F 1989 The synthesis of $\mathrm{AlPO}_{4}-20$, BAPO-20 and SAPO-20 molecular sieve $J$. Taiyuan Univ. Technol. 2021.

[5] Wilson S T, Lok B M and Flanigen E M 1982 Crystalline Metallophosphate Compositions US Patent 4310440.

[6] Kaiser S W, Charleston S and Va W 1987 Production of Light Olefins US Patent 4677242.

[7] Li H Y, Liang J, Liu Z M, Zhao S Q and Wang R H 1988 Synthesis of silicoaluminophosphate zeolites SAPO-11, SAPO-34 and SAPO-20 Chinese J. Catal. 9503.

[8] Dumitriu E, Azzouz A, Hulea V and Lutic D 1997 Synthesis, characterization and catalytic activity of SAPO-34 obtained with piperidine as templating agent Micropor. Mater. 101.

[9] Sunil A, Chilukuri S V V, Prakash A M and Chakrabarty D K 1997 Small pore aluminum phosphate molecular sieves with chabazite structure: Incorporation of manganese in the structures-34 and $-44 J$. Phys .Chem. 1003665.

[10] Hasha D, Saldarriaga L S D, Saldarriaga C, Hathaway P E, Cox D F and Davis M E 1988 Studies of silicoaluminophosphates with the sodalite structure J. Am. Chem. Soc.110 2127.

[11] Kuehl G H and Cherry H N J 1988 Preparation of Crystalline Silicoaluminophosphates US Patent 4786487.

[12] Zhao D Q, Qiu S L, Pang W Q, Zhang J G, Wu Y J and Pei F K 1992 Studies on the synthesis of molecular sieves from TMAOH- $\mathrm{P}_{2} \mathrm{O}_{5}-\mathrm{Al}_{2} \mathrm{O}_{3}-\mathrm{SiO}_{2}-\mathrm{H}_{2} \mathrm{O}-\mathrm{HF}$ system Chem. Res. Chin. Univ. 13717.

[13] Bandyopadhyay M, Bandyopadhyay R, Tawada S, Kubota Y and Sugi Y 2002 Catalytic performance of silicoaluminophosphate (SAPO) molecular sieves in the isopropylation of biphenyl Appl. Catal. A 225 51.

[14] Wang X M 2008 Study on the Synthesis of SAPO Molecular Sieve with Liquid-Phase Crystallization Method (ShanXi: Northwest University press) p 43.

[15] Itakura M, Ota K, Shibata S, Inoue T, Sadakane M and Sano T 2011 Influence of starting zeolite on synthesis of RUT type zeolite by interzeolite conversion method J. Cryst. Growth 314274.

[16] Yamanaka N, Itakura M, Kiyozumi Y, Ide Y, Sadakane M and Sano T 2012 Acid stability evaluation of CHA-type zeolites synthesized by interzeolite conversion of FAU-type zeolite and their membrane 
application for dehydration of acetic acid aqueous solution Micropor. Mesopor. Mater. 158141.

[17] Shibata S, Itakura M, Ide Y, Sadakane M and Sano T 2011 FAU-LEV interzeolite conversion in fluoride media Micropor. Mesopor. Mater. 13832.

[18] Itakura M, Goto I, Takahashi A, Fujitani T, Ide Y, Sadakane M and Sano T 2011 Synthesis of high-silica CHA type zeolite by interzeolite conversion of FAU type zeolite in the presence of seed crystals Micropor. Mesopor. Mater. 14491.

[19] Zones S I 1990 Direct hydrothermal conversion of cubic P zeolite to organozeolite SSZ-13 J. Chem. Soc. Faraday Trans. 863467.

[20] Zhang HR, Zhang Q, Li YP, Zhao C and Dou T 2010 Direct synthesis and methanol conversion catalytic performance of PZSM-5 zeolite with preformed SAPO-34 molecular sieve Acta Petrolei Sinica (Petroleum Processing Section) 26357

[21] Lee Y J, Baek S C and Jun K W 2007 Methanol conversion on SAPO-34 catalysts prepared by mixed template method Appl. Catal. A 329130. 